\title{
Ein österreichischer Zola? Zu Jakob Julius Davids «Wiener Romanen »
}

Un Zola autrichien ? Les « romans viennois » de Jakob Julius David An Austrian Zola? The "Viennese Novels" of Jakob Julius David

\section{Martin Erian}

\section{OpenEdition}

\section{Journals}

Édition électronique

URL : http://journals.openedition.org/austriaca/600

DOI : 10.4000 /austriaca.600

ISSN : 2729-0603

Éditeur

Presses universitaires de Rouen et du Havre

Édition imprimée

Date de publication : 1 juin 2018

Pagination : 149-164

ISBN : 979-10-240-1233-9

ISSN : 0396-4590

\section{Référence électronique}

Martin Erian, "Ein österreichischer Zola? Zu Jakob Julius Davids « Wiener Romanen ", Austriaca

[Online], 86 | 2018, Online erschienen am: 01 Juli 2020, abgerufen am 29 Januar 2021. URL: http:// journals.openedition.org/austriaca/600 ; DOI: https://doi.org/10.4000/austriaca.600 
Martin ERIAN

Université de Klagenfurt

\section{Ein österreichischer Zola? $\mathrm{Zu}$ Jakob Julius Davids «Wiener Romanen»}

\section{Das lange Warten auf ,den Wiener Roman}

Die Umgestaltung in der zweiten Hälfte des 19. Jahrhunderts, die vor allem mit der Schleifung der Stadtmauern, dem Bau der Ringstraße sowie der Eingemeindung der Vorstädte der Residenzstadt an der Donau ein neues Gesicht verlieh und mit enormem Zuzug einherging, machte Wien nicht nur zur Zwei-Millionen-Einwohner-Metropole. Sie brachte gemeinsam mit der Modernisierung auch die materiellen und kognitiven Voraussetzungen des urbanen Zusammenlebens ins Wanken und änderte die Vorstellung der kleinstädtischen, de facto dörflichen Idee der Stadt zu jener des unübersichtlichen Molochs mit einer weitgehend hermetisch abgeriegelten sittlich-mentalen Kartographie. ${ }^{1}$ Der dem realistischen Schreiben verpflichtete Literaturbetrieb im Schatten der Wiener Moderne setzte sich anhaltend mit diesem urbanen Wandel auseinander - und ließ die Kritiker doch immer wieder vergeblich auf „de[n] eigentliche[n] Wiener Roman, nach dem schon Jahre und Jahre das Verlangen aller Literaturfreunde geht“2, warten. Selbst in ihrer verglichen mit anderen europäischen Großstädten beschränkten

1. Einführend dazu Wolfgang Maderthaner, „Von der Zeit um 1860 bis zum Jahr 1945“, in Peter Csendes, Ferdinand Opll (Hg.), Wien. Geschichte einer Stadt. Bd. 3: Von 1790 bis zur Gegenwart, Wien [u.a.], Böhlau, 2006, S. 175-544; Lutz Musner, „Stadt. Masse. Weib. Metropolenwandel, Massenphobie und Misogynie im Finde-Siècle“, in Günther Hödl (Hg.), Frauen in der Stadt, Linz (= Beiträge zur Geschichte der Städte Mitteleuropas, Bd. XVIII), Österreichischer Arbeitskreis für Stadtgeschichtsforschung, 2003, S. 63-81, hier S. 63 f.

2. N.N., „Literarische Kreuz- und Querzüge“, Badener Zeitung, 2.3.1907, S. 1-2, hier S. 1. 
Dynamik schien sich die habsburgische Metropole einer umfassenden Beschreibung zu entziehen:

Die österreichischen Erzähler größeren Stils schleichen um die Hauptstadt herum wie um ein verbotenes Futter. Sind die Probleme zu groß, die hier liegen? Widerstreben sie der Gestaltung? Oder ist das Ganze dieser Stadt zu unfaßbar, zu widerspruchsvoll, zu reich an heiklen Rücken und Tücken? [...] Und doch fühlt, wer in dieser Stadt lebt, den großen epischen Zug, der ihren Riesenkörper durchbebt, den ungeheuren Wachstums- und Umwandlungsprozeß, der sich aus dem friedlichen Dämmer der Stuben hinaus in den Lärm der Straßen drängt und der dann weiterflutet in die Hallen der Oeffentlichkeit, wo mächtige Gegensätze aufeinanderprallen. ${ }^{3}$

Rudolf Holzer strich beim Erscheinen Fritz Stüber-Gunthers Studentenroman C. i. (Cum infamia, 1907) den Mangel eines „alle Elemente des lebenatmenden [!], historisch entwickelnden, soziologisch erklärenden Wiener Romanes", also eines Textes nach Vorbild des europäischen Großstadtromans des späten 19. Jahrhunderts hervor und präzisierte: „Der Wiener Roman liegt in der Kunst der feinen Kleinschilderung, der minutiösen Echtheit der kleinen Leute. “4

Als ein maßgeblicher Vertreter des Genres konnte sich zur Jahrhundertwende Jakob Julius David hervortun, „auf ihm“, so schrieb Max Foges, sein langjähriger Kollege in der Redaktion des Neuen Wiener Journals in einem Nachruf, beruhten die Hoffnungen, dass er „es sein werde, der den österreichischen, den Wiener Roman in jener Vollendung der Literatur schenken würde, nach der bisher so viele schwächere Talente erfolglos strebten" 5 .

1859 in Mährisch-Weißkirchen als Sohn eines Mautpächters geboren, übersiedelte David im Herbst 1877 nach Wien, wo er mit dem Ziel einer Schriftstellerkarriere ${ }^{6}$ Deutsche Philologie bei Richard Heinzel und Erich Schmidt studierte. In deutschnationalen Kreisen fand der mährische Jude trotz mangelnder Studienerfolge - „[D]er Unterricht

3. Franz Servaes, „Neue österreichische Romane“, Neue Freie Presse, 18.11.1906, S. 32-35, hier S. 32.

4. Rudolf Holzer, „,C. i.' Roman von Fritz Stüber-Gunther“, Wiener Abendpost. Beilage zur Wiener Zeitung, 1.5.1907, S. 5. Die bisher unaufgearbeitete Debatte trieb weitere Blüten: Angeregt vom Vorwurf des gänzlichen Fehlens eines Wiener Romans veröffentlichte der Schriftsteller Albin Schanil eine Auflistung einschlägiger Texte der letzten fünfzig Jahre. Vgl. N.N., „Theater, Literatur und Kunst“, Wiener Abendpost. Beilage zur Wiener Zeitung, 25.9.1907, S. 2.

5. Max Foges, „J. J. David †“, Neues Wiener Journal, 21.11.1906, S. 1-2, hier S. 1.

6. Von dieser Absicht berichtete später sein Lehrer Hermann Hoffmann. Vgl. H.H., „J. J. David“, Badener Zeitung, 18.3.1908, S. 2-4, hier S. 3. 
[...] glitt halbvernommen an ihm vorbei“, erinnerte sich Schmidt, später Mitherausgeber der Gesammelten Werke ${ }^{7}$ - und monatelanger Obdachlosigkeit Anschluss und trat als kneipender Student und gefürchteter Billardspieler und Fechter in Erscheinung. ${ }^{8}$ Schmidts Bericht über das lange verheimlichte Leid des Studenten überrascht, ist in seinem Werk doch die, um mit Florian Krobb zu sprechen, „Tendenz, seinen Lebensweg als Leidensweg zu verklären, [...] unübersehbar"9. Dies gilt insbesondere für die im urbanen Raum angesiedelten Texte, aber auch für die Reflexion seiner sozialen wie schriftstellerischen Stellung. Von den Jung-Wienern um Hermann Bahr ausgeschlossen, ${ }^{10}$ schrieb David 1900 an Hugo Salus: „Es steht hier in Wien gegen mich eine Phalanx, gegen die ich nicht aufkomme, besonders nachdem ich keine Kampfnatur bin. "11

7. Erich Schmidt, „Vorwort“ zu Jakob Julius David, Gesammelte Werke. Bd. 1. Hg. von Ernst Heilborn und E. S., München, Leipzig, Piper, 1908, S. V-XXIII, hier S. XI. Weitere Zitate aus den Gesammelten Werken abgekürzt mit der Sigle GW inkl. Bandangabe. Die unvollständige Werksammlung - mehrere Texte, insbesondere publizistische Arbeiten, etwa die Feuilletons im Neuen Wiener Journal oder die späten Beiträge für die Wiener Abendpost, fanden bislang, folgenschwer für die David-Forschung, keine Berücksichtigung - musste herbe Kritik über sich ergehen lassen, namentlich Schmidt für sein Vorwort. Der Schriftsteller und Feuilletonist Franz Schamann nannte es ,eine Beleidigung dessen, dem sie das ,bleibende Gedächtnismal' schmücken sollte“. - F. S., „J. J. David“, Arbeiter-Zeitung, 14.4.1908, S. 1-3, hier S. 1.

8. Vgl. Schmidt, „Vorwort“, a.a.O., S. XI-XIII; Ella Spiero, Jakob Julius David, Leipzig, Finck, 1920, S. 21ff; Jakob Julius David, „Im Spiegel. Autobiographische Skizze“, Das literarische Echo 4 (1902), H. 8, S. 528-530, zitiert nach Florian Krobb, „Empfänglichkeit fürs Leid“, Nachwort zu: Jakob Julius David, Verstörte Zeit. Erzählungen. Hg. von F. K., Göttingen, Wallstein, 1990, S. 306-309.

9. Krobb, „Empfänglichkeit“, a.a.O., S. 314.

10. „Den David lassen wir weit, weit, weit, weit draußen“, schrieb Bahr 1896 an Franz Servaes, nachdem David um 1890 noch zu den häufigsten Beiträgern in der Zeitschrift Moderne Dichtung/Moderne Rundschau gezählt hatte. Zitiert nach Peter Sprengel, Gregor Streim, Berliner und Wiener Moderne. Vermittlungen und Abgrenzungen in Literatur, Theater, Publizistik, Wien (= Literatur in der Geschichte, Geschichte in der Literatur, Bd. 45), Wien [u.a.], Böhlau, 1998, S. 105. Später schrieb David anonym über sein Werk, „schroff für sich allein zu stehen“. - Nemo [= J. J. David], „Assimilation“, Dr. Bloch's Oesterreichische Wochenschrift, 7.5.1897, S. 390-391, hier S. 391.

11. Jakob Julius David an Hugo Salus, 1.8.1900, zitiert nach Herman Groeneweg, J. J. David in seinem Verhältnis zur Heimat, Geschichte, Gesellschaft und Literatur, Graz (= Deutsche Quellen und Studien, Bd. 4), Stiasny, 1929, S. 194. Dass Davids Werk „merkwürdig quer“ (Michael Worbs) zur Literatur der Jahrhundertwende steht und damit dem Vergessen preisgegeben ist, wurde mit den Mechanismen der Literaturgeschichtsschreibung bis vor Kurzem einzementiert. Vgl. Clemens Peck, „Paralysis progressiva'. Zur Figuration des Bildungsproletariats in Jakob 


\section{„Nur ein Späher“ - David als Lyriker und Journalist in der Großstadt}

Davids Begegnungen mit der Metropole Wien waren ebenso vom Schicksal des Zuwanderers wie von jenem des intellektuellen Proletariers geprägt. Nach Anstellungen als Hauslehrer war er bis zur gesundheitlich bedingten Aufgabe als Journalist tätig, die Stelle als Schriftleiter beim Neuen Wiener Journal zwischen 1894 und 1903 sicherte ihm 200 Gulden monatlich. ${ }^{12}$ Seine Geltung als Publizist half wohl dabei, seinen literarischen Arbeiten Publikationsräume zu eröffnen, wenn er auch zeitlebens mit seiner Doppelrolle haderte. ${ }^{13}$ In seiner Studie Die Zeitung, 1906 in der von Martin Buber verantworteten Reihe Die Gesellschaft erschienen, präsentiert David das Journalistendasein als eine prototypische Rolle des ökonomisch unversorgten Universitätsabsolventen in der Großstadt, konnte das Zeitungswesen sein Personal doch günstig aus „dem unermeßlichem [sic!] Reservoir des gebildeten Proletariats, das zunächst einmal froh sein muß, überhaupt eine Verwendung für sich und seine Kenntnisse zu finden"14, rekrutieren. Bereits Davids erste Erzählung, „Fanny“, 1886 in der Deutschen Zeitung publiziert und 1890 unter dem Titel Das Höferecht als Buch verlegt, deutet auf das Elend junger Redakteure hin; die 1892 in der Sammlung Probleme veröffentlichte und Ende des Jahres auch im Feuilleton der Arbeiter-Zeitung abgedruckte

Julius Davids Wien-Roman ,Am Wege sterben“", Internationales Archiv für Sozialgeschichte der deutschen Literatur 35 (2010), H. 2, S. 37-60, hier S. 37-41.

12. Vgl. Konrad Paul Liessmann, „Jakob Julius David und die Kunst der Novelle im Fin de siècle“, in J. J. David, Novellen, Salzburg, Wien (= Eine österreichische Bibliothek), Residenz, 1995, S. 259-291, hier S. 264.

13. Vgl. David, „Im Spiegel ...“, a.a.O., S. 308. So klagte Stefan Zweig, dass man „einen solchen Dichter die besten Jahre in journalistischer Tretmühle verkommen ließ“. Karl Kraus ätzte, „daß es nur in dieser maßlosesten Stadt der Welt möglich ist, einen J. J. David ein Leben im Dienste des Herrn Lippowitz [Jakob L., Chefredakteur des Neuen Wiener Journals, Anm.] führen zu lassen. - Stefan Zweig, „Dem Gedächtnis J. J. Davids“, Österreichische Rundschau (1906), H. 9, S. 217-219, hier S. 219; Karl Kraus, „Antworten des Herausgebers“, Die Fackel VIII (1907), Nr. 221, S. 17.

14. Jakob Julius David, Die Zeitung. Frankfurt/M. (= Die Gesellschaft. Sammlung sozialpsychologischer Monographien, Bd. 5), Literarische Anstalt Rütten \& Loening, 1906, S. 42. 
Erzählung „Ein Poet?“ zeigt das erst im Selbstmord aufgelöste Dilemma zwischen dichterischen Ambitionen und journalistischem Brotberuf. ${ }^{15}$

$\mathrm{Zu}$ diesem Zeitpunkt hatte sich David bereits als Lyriker einen Namen gemacht, wenngleich die Sammlung Gedichte ebenfalls erst 1892 erschien. ${ }^{16}$ Seine Lieder von der Straße waren im Herbst 1891 der Höhepunkt einer wenig erfolgreichen Veranstaltung der Neuen Freien Bühne um Friedrich M. Fels in den Wiener Sofiensälen. ${ }^{17}$ Sie beinhalten deutlich den angedeuteten larmoyanten Ton; die Not fungiere nicht nur als Muse, sondern sei die beste Freundin des gepeinigten lyrischen Ichs. ${ }^{18}$ Ludwig Speidel, Anhänger Davids "gehämmerte[r], persönliche[r] Prosa“, urteilte: „Wie es die Art der Modernen ist, beschäftigt er [David, Anm.] sich viel mit sich selbst, oft interessant genug, aber nicht immer das Maß des guten Geschmacks einhaltend. Mit Klagen über vergangene Not muß man haushalten. " ${ }^{19}$ Zugleich zeugen die Lieder von der Straße in programmatischen Äußerungen von der Parteinahme des sozialkritischen, selbst aus der Oberschicht ausgeschlossenen Autors $^{20}$ und seinem Verständnis für Krisenerfahrungen der Moderne. Das die Sammlung beschließende Gedicht „Im Volkston“ („Ich hab“

15. Vgl. dazu Jürgen Doll, „Mourir en chemin: presse et littérature chez Jakob Julius David (1859-1906)“, in Jacques Le Rider, René Wentzig (Hg.), „Les journalistes“ de Arthur Schnitzler. Satire de la presse et des journalistes dans le théâtre allemand et autrichien contemporain, Tusson (= Transferts), Du Lérot, 1995, S. 103-117.

16. Bereits 1890 griff Emil Reich Davids bis dato ausschließlich verstreut erschienene Gedichte in einer Kritik auf, der Band Gedichte wurde überwiegend positiv rezensiert. - Vgl. Emil Reich, „Oesterreichische Lyriker“, Wiener Zeitung, 19.6.1890, S. 2-3; d. m., „Neue Gedichte“, Allgemeine Kunst-Chronik XVI (1892), Nr. 2, S. 50; Bruno Walden, „Poesie“, Wiener Abendpost. Beilage zur Wiener Zeitung, 16.1.1892, S. 1-2.

17. Vgl. L., „Die freie Bühne“, Allgemeine Kunst-Chronik XV (1891), Nr. 23, S. 635.

18. Vgl. „Noth“ und „Meine Muse“, in Jakob Julius David, Gedichte, Dresden, Leipzig, Minden, 1892, S. 4, 41.

19. Ludwig Speidel, „Drei Lyriker“, Neue Freie Presse, 2.4.1893, S. 1-2, hier S. 2.

20. „Ich habe wenig mit dem Geistesadel, minder noch mit dem der Geburt zu thun“, schrieb David wohl Anfang 1889 an Marie von Ebner-Eschenbach, zitiert nach Jiří Veselý, „Ebner-Eschenbach - Saar - David. Tschechische Elemente in ihrem Werk und Leben“, Lenau Forum 1 (1969), S. 25-45, hier S. 31. Auch vom lyrischen Praterspaziergang bleibt bloß der Grimm über die soziale Not: „Das Leid der Armen, Verderbten/Erstand mir qualvoll -/Indes der Haß des Enterbten/In meiner Seele quoll.“ - Jakob Julius David, „Sonntag“, in David, Gedichte, a.a.O., S. 44f., hier S. 45. Zur "Schnittstelle Prater" siehe auch Moritz Csáky, Das Gedächtnis der Städte. Kulturelle Verflechtungen - Wien und die urbanen Milieus in Zentraleuropa, Wien [u.a.], Böhlau, 2010, S. 208-217. 
kein' Hof, ich hab' kein Feld,/Ich hab' kein' Heimath in der Welt“21) macht auf die ausbleibende Assimilation aufmerksam, ohne dabei den städtischen Raum explizit als seinen Schauplatz zu benennen; allerdings greift es mit dem Hinweis auf die völlige Entwurzelung des Individuums einen zentralen thematischen Marker der Urbanitätserfahrung um 1900 auf. Wenn es also in „Meine Muse“ heißt „Ich bin kein Dichter, nur ein Späher,/Ich hör', was unterirdisch kocht,/Wie schwielenfäustig der Plebejer/An der Paläste Pforten pocht" ${ }^{\text {"22 }}$, so umreißt dies nicht nur das Selbstverständnis des Autors, sondern ist auch ein Einblick in sein Themenrepertoire. Hier paraphrasiert David bereits das Schicksal des studentischen Proletariers Karl Stara im Roman Am Wege sterben, den schon eingangs „ein ingrimmiger Haß gegen diese Stadt" erfüllt, droht ihm doch das Schicksal, „wie ein ausgestoßener Hund an der Schwelle dieser Paläste ${ }^{\text {“23 }}$ zu verenden.

\section{Am Wege sterben - Studentische Sozialexpeditionen ohne glückliches Ende}

Auch wenn David in den Neunzigerjahren zunächst vorwiegend historische Novellen schrieb, dürfte die Auseinandersetzung mit dem urbanen Raum in der epischen Großform ein langgehegtes Ziel des Autors gewesen sein. Bereits am 18. März 1889 hielt David im Wiener wissenschaftlichen Club einen Vortrag über den „modernen Wiener Roman “; 24 in seinem Essay zum hundertsten Geburtstag Franz Grillparzers 1891 adelte er dessen Erzählung Der arme Spielmann als den Wiener Roman, der alle „Forderungen der jungen Schule an solch ein Werk“ erfülle, „und zwar in so großer Vollendung, daß man zweifeln muß, ob all dem leidenschaftlichen Bemühen unserer Tage etwas gelingen werde, wie es höchster Kunstverstand und genaueste Kenntnis des Stoffes

21. Jakob Julius David, „Im Volkston“, in David, Gedichte, a.a.O., S. 52.1903 fand das Gedicht Aufnahme in die Sammlung Lieder aus dem Rinnstein Hans Ostwalds, Herausgeber der Großstadt-Dokumente. Vgl. H. O. (Hg.), Lieder aus dem Rinnstein. Erstes Bändchen, Leipzig, Berlin, Henckell, 1903, S. 98.

22. David, „Muse,“ a.a.O., S. 41.

23. Jakob Julius David, Am Wege sterben, in J. J. D., GW, Bd. 4, a.a.O., S. 103-316, hier S. 117. Weitere Zitate werden mit der Sigle AWS sowie der Seitenzahl im Haupttext angegeben.

24. Vgl. N. N., „Vereinsnachrichten“, Wiener Zeitung, 17.3.1889, S. 4. 
anscheinend mühelos geschafft haben“25. Die frühe Erzählung Das Höferecht und der 1890 in Fortsetzungen in der Neuen Freien Presse und 1891 als Buch veröffentlichte Roman Das Blut, denen überwiegend das Dorf als Bühne dient, blicken in Richtung Sündenpfuhl Großstadt, auch die Dramen Ein Regentag und Neigung bedienen sich Wiener Typen. Im Vorwort zu Ein Regentag konstatierte David: „[I]ch glaube selber, daß ich an manche fressende und schwärende Wunde des Wiener Lebens mit nicht sehr milden Fingern gerührt. ${ }^{26}$ Ab 6. August 1899 erschien schließlich der - so die Ankündigung - „Wiener Original-Roman“ Am Wege sterben in 34 Folgen in der Neuen Freien Presse.

Clemens Peck hat aus gutem Grund den Experimentalcharakter des Werks hervorgestrichen, knüpft David doch deutlich an Émile Zolas seit den Achtzigerjahren breit diskutiertes Konzept des roman expérimental an, nach dem der „Romanschriftsteller aus einem Beobachter und einem Experimentator besteht ${ }^{\text {‘27. }}$. Der in Davids Studentenzeit angesiedelte Roman lässt sich auch als literarische Reportage lesen, unternehmen die Protagonisten, vier Studenten, doch Expeditionen in vermeintlich undurchlässige Milieus, ob in die Zinshäuser oder die innerstädtischen Salons, innerhalb des habsburgischen Schmelztiegels Wien. Auch die Neue Freie Presse hob in einer Besprechung neben der „dokumentarische[n] Treue“ den „ethnologischen Reiz" des Werks hervor, biete es doch „einen Querschnitt durch die verschiedenen Bevölkerungsschichten, wie sie in den verschiedenen Stockwerken der großen Häuser, aber auch in den verschiedenen Bezirken der großen Stadt getrennt

25. Jakob Julius David, „Franz Grillparzer“, in J. J. D., GW, Bd. 7, a.a.O., S. 1-17, hier S. $15 \mathrm{f}$.

26. Jakob Julius David, „Vorwort“ zu „Ein Regentag“, in J. J. D., GW, Bd. 3, a.a.O., S. 193-195, hier S. 194.

27. Émile Zola, Der Experimentalroman. Eine Studie. Autorisierte Übertragung von Julius Zeitler, Leipzig, Zeitler, 1904, S. 11-62, hier S. 13. Vgl. Peck, „Paralysis ...“, a.a.O., S. 42. Zur Zola-Rezeption siehe u. a. Manfred Brauneck, Christine Müller (Hg.), Naturalismus. Manifeste und Dokumente zur deutschen Literatur 1880-1900, Stuttgart, Metzler, 1987, S. 646-736; Jacques Le Rider, „Naturalismus in bleu-blancrouge, Schwarz-Weiß-Rot und Schwarzgelb", in Roland Innerhofer, Daniela Strigl (Hg.), Sonderweg in Schwarzgelb? Auf der Suche nach einem österreichischen Naturalismus in der Literatur, Innsbruck, StudienVerlag, 2016, S. 33-56; Karl Zieger, Die Aufnahme der Werke von Émile Zola durch die österreichische Literaturkritik der Jahrhundertwende, Bern [u.a.] (= Europäische Hochschulschriften: Reihe 18, Vergleichende Literaturwissenschaft, Bd. 44), Lang, 1986. 
werden“28. Im Gegenzug erscheint die Zuschreibung des „typische[n] Großstadt-Studentenroman [s] "29 durchaus als fragwürdig, dienen etwa Karl Hans Strobls ab 1902 erschienene Prager Studentenromane Die Vaclavbude, Der Schipkapaß und Das Wirtshaus "Zum König Przemysl" als Vergleichsfolien. So spielt jene von Karl-Markus Gauß Davids Text

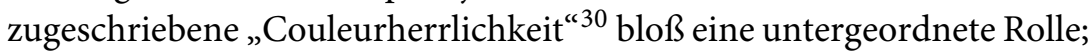
Am Wege sterben stützte zeitgenössische Debatten um fehlende studentische Kultur in Wien. ${ }^{31}$

Der Roman verhandelt in zwei Büchern die Schicksale jener vier Studenten, die in einem abseitig gelegenen Gasthaus in der Josefstadt den Ausgangspunkt ihrer Wege durch die sozialen Topographien des Wiener Lebens finden und in der Hoffnung auf den persönlichen Aufstieg in der Stadt durch Studien- oder Liebeserfolge das häufig tragische Los der Zugezogenen ziehen. Das ,Delirium tremens', wie sie das Lokal nennen, besitzt verschiedentlich die Funktion der kulturellen Schnittstelle: ${ }^{32}$ als studentischer Treffpunkt, als Knotenpunkt der Gesellschaftsschichten und nicht zuletzt als sicherer Hafen der Zugewanderten, auch wenn er ein mahnendes Faktotum aufweist: Franz Kubat, mit großen Ambitionen einst nach Wien gekommener Medizinstudent, nun 48-jähriger Alkoholiker. Als der selbsternannte „Studentenvater“(AWS, 208) Clemens Deym seine Wirtschaft für immer schließen möchte, erwürgt Kubat ihn im Wahn. ${ }^{33}$

28. Gz., „Literarische Notizen“, Neue Freie Presse, 25.3.1900, S. 33. Der Roman wurde mehrfach ausdrücklich positiv rezensiert. Vgl. auch N.N., „Ein neuer Roman“, Prager Tagblatt, 23.3.1900, S. 1; Max Foges, „Wiener Dichter (Jakob Julius David, Balduin Groller, Ferdinand Groß.)", Neues Wiener Journal, 30.4.1900, S. 1-2.

29. Oskar F. Scheuer, „Der Wiener Student im Roman“, Neues Wiener Tagblatt, 19.5.1926, S. 1-4, hier S. 2.

30. Karl-Markus Gauß, „Jakob Julius David oder Er starb am Wege“, in K.-M. G., Tinte ist bitter. Literarische Porträts aus Barbaropa, 2. Auflage, Klagenfurt, Wieser, 1992, S. 149-159, hier S. 154.

31. Vgl. Alfred Gold, „Studentenromantik“, in Die Zeit, 2.6.190o, S. 138-139; Otto Kraus, „Die jüngsten Wiener“, in Agramer Zeitung, 16.6.1900, S. 1of. Auch die von Giovanni Tateo vorgenommene Charakterisierung des Romans als ,angewidertes Fresko des Studentenlebens" scheint nur insofern angemessen, als dass der Roman auf die den universitären Alltag überlagernden sozialen Nöte der Studenten hinweist. - G. T., „Zwischen Hauptstadt und mährischer Provinz. Jakob Julius Davids Erzählung Die Hanna“, in Studia Germanica Posnaniensia XXXII (2011), S. 121-135, hier S. 125.

32. Vgl. dazu auch Csáky, Gedächtnis, a.a.O., S. 149-153.

33. In Vorstufen bildete Kubat noch die Hauptfigur des Textes. Vgl. Franz Schamann, „Aus J. J. Davids Nachlaß“, in Österreichische Rundschau XXIII (1910), S. 429. 
Dieser das zweite Buch eröffnende Mordfall löst die soziale Klammer, das individuelle Scheitern beginnt. Während der von zweifelhafter Schneid ??(Mut)gekennzeichnete (wohl Michael Georg Conrad nachempfundene ${ }^{34}$ ) Wiener Eduard Beyerl nach zwischenzeitlichem Abdriften in Trunksucht und Kriminalität noch ein Auslangen als kleiner Beamter findet, erleiden Stara und Raimund Förster Schiffbruch. Stara, „fremd und mittellos“(AWS, 111) aus Mähren zugewandert, besitzt aus Angst, zu kurz zu kommen, eine bis zur Selbstverleugnung reichende Anpassungsfähigkeit (AWS, 114). Ursprünglich für das Priesteramt vorgesehen und nun „billigen Liebensabenteuern“ (AWS, 189) im Prater nicht abhold, erhält er als Hauslehrer Zutritt zur Oberschicht und strebt wie sein Dienstgeber den sozialen Aufstieg durch Heirat an. „Man sah, er bereitete sich wieder zu einem großen Sprunge. ,Der Kerl pomadisiert sich und spricht schon beinahe wie ein Hofrat,' urteilte Beyerl. Etwas Schlimmeres hat er nie jemandem nachgesagt." (AWS, 223) Doch sein Plan, die adelige Hofratstochter zu seinem Steigbügel zu formen, platzt, frustriert treibt er stattdessen die junge Proletarierin Resi, Tochter seiner Zimmerfrau, mit überhandnehmenden Zudringlichkeiten in den Tod. Er selbst verwirft die Idee des Suizids und beklagt im Gegenzug die Haltung seiner Familie, die ihn zum Studium in die Stadt geschickt hat:

[W]as gingen ihn die Seinen an, deren dummer Ehrgeiz, einen Gelehrten durchaus in der Familie haben zu wollen, allein dies sein beklagenswertes Ende verschuldet! Hätt' er den Grund übernommen und heiraten können, so säß' er nun wohl und warm daheim. (AWS, 306)

Er endet, Ausdruck des Klischees vom listigen Böhmen, als Polizeispitzel.

Förster verbleibt Nebenfigur, hat jedoch das härteste Schicksal zu tragen, dessen Darstellung David wiederholt beschäftigte. „Er war gar $\mathrm{zu}$ arm von Hause und ist vor lauter Hunger nicht zum Studieren gekommen “35, wird sein Schicksal bereits in „Ein Poet?“ skizziert. In Am Wege sterben tritt er von Beginn an als Geschlagener auf, obdachlos, unangepasst und von offensichtlicher körperlicher Schwäche. Als einzigen Besitz trägt er, der aus „Ekel über sein verpfuschtes Sein“ (AWS, 136) immer wieder sein Hab und Gut verliert und der Gelegenheit, ein

34. Vgl. Clemens Peck, „Jakob Julius Davids Naturalismen“, in Roland Innerhofer, Daniela Strigl (Hg.), Sonderweg in Schwarzgelb? Auf der Suche nach einem österreichischen Naturalismus in der Literatur, Innsbruck, Studienverlag, 2016, S. 153-168, hier S. 162.

35. Jakob Julius David, „Ein Poet?“, in J. J. D., GW, Bd. 3, a.a.O., S. 152-190, hier S. 164. 
Handwerk zu erlernen, nachtrauert, einen Vogel durch die Straßen Wiens. „[I]ch hab' sonst nichts von zu Hause. Und wenn er anfängt zu schlagen, so seh' ich Hohenolbersdorf [...], und das tut mir so gut in den Augen." (AWS, 108) Die Entwurzelung sollte ihm nicht gelingen. Seine Erkenntnis aus „Ein Poet?“ einlösend - „[H]eut, hab' ich Napoleon an der Moskwa verstanden. Man opfert nicht so weit von der Heimat seine letzten Reserven " 36 -, kehrt der einst herausragende Zögling nach Schlesien zurück, um 28-jährig zu sterben. „Vielleicht erschrickt einer, wenn er mich sieht, als eine Warnung, und geht nicht meinen Weg, und ich bin doch noch was nutz gewesen auf der Welt.“ (AWS, 251)

Einzig der Jude Simon Siebenschein kann spät seine Studien beenden. Er wird auf den Spuren Viktor Adlers, Marie von Ebner-Eschenbachs Kreisphysikus Nathanael Rosenzweig, aber auch Zolas Docteur Pascal ${ }^{37}$ Armenarzt in Ottakring, schon als Pfleger des gelähmten Sohnes seines verarmten Vermieters dient er als Hoffnungsträger. Zum positiven Gegenentwurf seiner Kommilitonen reicht es freilich nicht: Gebrandmarkt „,on dem Hochmute seiner Race“ (AWS, 145), einer befremdlichen, den zeitgenössischen antisemitischen Diskursen verpflichteten Körperlichkeit und dem zeitweisen Luxusbedürfnis des „durch Bildung überfeinerten Menschen“ (AWS, 313), scheut er als „schamlose[r] Weltverächter" (AWS, 199) jeden soziale Grenzen überschreitenden Kontakt. Weder die Liebesnacht mit einer im Prater eroberten Proletarierin rührt sein Herz („Derlei mußte getrunken werden in raschem Zuge [...].“ - AWS, 202), noch sein späteres Wirken als sozialistisch inspirierter Armenarzt. Siebenschein, der vor Resis Leichnam auch an seinem eigenen Weg zweifelt („Aber wozu ist alles auf der Welt? Der Verstand? [...] Das Wissen? [...] Die Schönheit? Da schau her: hab nichts Schöneres gekannt, und sieh dir sie nur an, wie sie jetzt daliegt.“- AWS, 286) bleibt unnahbarer Individualist ${ }^{38}$ und er spricht das Fazit für sich und die "ganze Phalanx von Morituri“" ${ }^{39}$ des Romans: „Wir haben alle zu schwer am Wege gelitten. [...] Wir sind alle verlorene Söhne mit oder ohne Titel und Amt. Wir haben keinen Glauben mehr, und zu uns hat ihn keine Menschenseele mehr." (AWS, 311).

36. Ebd., S. 165.

37. Vgl. Peck, „Paralysis ...“, a.a.O., S. 47-56.

38. So ist die von Peck attestierte Überwindung der „Probleme der jüdischen Emanzipation durch soziales Engagement" gewiss keine vollständige. Vgl. Peck, „Paralysis ...", a.a.O., S. 52; siehe zu Siebenschein auch Peck, „Naturalismen ...“, a.a.O., S. 163-165.

39. N. N., „Roman“, a.a.O., S. 1. 


\section{Der Übergang: ,Moraldarwinistischer' Abgesang mit Ausstiegsmöglichkeiten}

Davids zweiter Wiener Roman Der Übergang erschien wischen 22. Jänner und 13. März 1903 in 44 Fortsetzungen in der Zeitung Die Zeit und lag bis vor Kurzem abseits der „kleinen Renaissance der David-Forschung " ${ }^{40}$, wurde er doch obwohl mehrfach neuaufgelegt und von Zeitgenossen als Höhepunkt von Davids Schaffen herausgehoben, ${ }^{41}$ wiederholt als mitunter triviales Werk ohne die treffende Zeitdiagnose der im Jahr zuvor veröffentlichten Buddenbrooks Thomas Manns abqualifiziert. ${ }^{42}$ Gauß deutete den Roman im Anschluss an Am Wege sterben als Rache der Zukurzgekommenen und als einen von Ideologie verdunkelten „Versuch, den lichten Sieg der Provinzler über die verkommenen Großstädter herbeizusehnen “43. Im Fokus steht jedoch der Niedergang einer Wiener Seidenfabrikantendynastie, der mit dem Wandel von patriarchalisch geprägten zu individualisierenden Lebensformen im späten 19. Jahrhundert und dem Ankommen der sozialen Frage im Bürgertum einhergeht. ${ }^{44}$ Zur Zeit des Wiener Kongresses scheint der „Wohlstand

40. Jörg Krappmann, Allerhand Übergänge. Interkulturelle Analysen der regionalen Literatur in Böhmen und Mähren sowie der deutschen Literatur in Prag (1890-1918), Bielefeld (= Interkulturalität, Bd. 4), transcript, 2013, S. 247.

41. Der Übergang sei Davids „stärkstes Können im Roman“ (Erich Schmidt), ihm gelinge „zum erstenmal ein ganz geschlossenes, einheitliches Kunstwerk“ (Stefan Hock), die „vornehme literarische Leistung“ zeichne „dieses gräßliche Nachtbild des Wiener sozialen Lebens mit zäher Folgerichtigkeit" (Marco Brociner). Vgl. Schmidt, "Vorwort“, a.a.O., S. XV; Stefan Hock, „David, Jakob Julius“, in Biographisches Jahrbuch und Deutscher Nekrolog XI (1908), S. 185-190, hier S. 189; Marco Brociner, „Der Uebergang“, Neues Wiener Tagblatt, 3.5.1903, S. 33. Der Übergang wurde u.a. 1910 in gekürzter Form „für den Schulgebrauch“, 1950 im Wiener Volksbuchverlag sowie in der DDR im Verlag Der Morgen und in der Reihe RomanZeitung des Verlags Volk und Welt neu aufgelegt.

42. Vgl. zuletzt Herbert Zeman, „Die österreichische Literatur an der Wende vom 19. zum 20. Jahrhundert“, in H. Z. (Hg.), Literaturgeschichte ÖsterreichS. Von den Anfängen im Mittelalter bis zur Gegenwart, 2. Auflage, Freiburg im Breisgau, Berlin, Wien, Rombach, 2014, S. 493-632, hier S. 559.

43. Gauß, „David“, a.a.O., S. 155.

44. Vgl. Krappmann, Übergänge ..., a.a.O., S. 256-263. Mit der Geschichte der (untergehenden) Seidenfabrikantendynastie vom Brillantengrund bedient sich David Wiener Typen, die es auch ins Wienerlied geschafft haben. Denn „unser Vater is a Hausherr und a Seidenfabrikant" lautet der Refrain von Johann Siolys und Wilhelm Wiesbergs um 1880 entstandenen Titel Die Hausherrnsöhnl'n. 
seines Hauses für immer begründet “45, als die heimatliche Gasse nach Firmengründer Adam Mayer sen. benannt wird. Doch es kommt anders. Als um das Krisenjahr 1873 das Wirtschaftsleben schnelllebiger wird, beginnt das Abrutschen der Dynastie, mittlerweile in zweiter Generation. „Die Mayerischen sahen verdrossen zu, und ihnen ward schwindelig von diesem Auf und Nieder. Das war keine Zeit, in die sie paßten."(ÜB, 181f) Der Roman ist nicht nur durch klischeebehaftete Typen und den Einsatz dialektaler Wendungen lokal grundiert, sondern insbesondere durch die Situierung - nimmt man Ausflugsfahrten aus - im Mikrokosmos der Schottenfelder Adam-Mayer-Gasse abseits des urbanen Treibens; ihre Beschreibung eröffnet den Text:

Die Gasse ist breit und ansehnlich genug. Nur sehr still ist sie. Man merkt in ihr wenig vom Verkehr und von der Betriebsamkeit, die sonst gerade in diesem Bezirk heimisch sind und sich immer noch behaupten.

Uniforme Häuser bilden sie: ein- oder höchstens zweistöckig. Ohne jeden Stil; man erkennt gleichzeitige Entstehung aus einem Willen. Aber sie sind tüchtig und für gute Dauer aufgemauert. Jedes hat einen tiefen Hof mit einigen Bäumen darin, die fröhlich gedeihen. Man wohnt wohlfeil da, und ein Wechsel der Parteien, wenn nicht eine völlig verdirbt, ist unerhört. (ÜB, 177)

Stillstand, Immobilität und fehlende Modernität bilden also die Kennzeichen der Gasse und des Habitus ihrer Bewohner („[W]ie Angehörige eines vereinzelten Stammes inmitten der Großstadt" - ÜB, 178) und deuten bereits zu Beginn auf die problematischen Perspektiven hin, stehen bei David doch wiederholt vormoderne Lebensformen unter einem schlechten Stern. Wie Wenzel Wondra in Am Wege sterben als verarmter Meerschaumpfeifendrechsler die Karikatur des Moderneverlierers mimt und die Anschauung entwickelt, wonach „aller Niedergang Wiens mit dem seines Handwerkes untrennbar zusammenhing" (AWS, 127) ${ }^{46}$ so

45. Jakob Julius David, „Der Übergang“, in J. J. D., GW, Bd. 5, S. 173-400, hier S. 18o. Weitere Zitate werden mit der Sigle ÜB sowie der Seitenzahl im Haupttext angegeben.

46. Vgl. Peck, „Paralysis ...“, a.a.O., S. 54f. Hierzu ist auch Davids Feuilleton „Ein Sonderling“ über einen eigenwilligen Hausbesitzer, „Einsiedler“ und „Traumwandler“ in einer stillen Gasse in Betracht zu ziehen, der ebenfalls mit Meerschaumpfeife auftritt. Ludwig Hirschfeld, ab 1906 Lokalfeuilletonist der Neuen Freien Presse, erhob Davids Meerschaumpfeifendrechsler später zum Topos, als Figur, die „die ganze Menschheit haßt und verachtet, weil sie vom Meerschaum nichts mehr wissen will und sich lieber der wohlfeilen papierenen Zigarrenspitzen bedient“. - J. J. D., „Ein Sonderling“, Neues Wiener Journal, 26.2.1895, S. 1-2; L. H., „Abschied vom Cobenzl“, Neue Freie Presse, 3.11.1907, S. 11. 
liegen in der Selbstinszenierung Franz Mayers, Patriarch in dritter Generation und Protagonist des Romans, die deutlichen Anzeichen seiner Unzeitgemäßheit. Er selbst fühlt, dass „dieser moderne Geist [...] ihm nichts Gutes" (ÜB, 183) bedeute:

Er ging noch manches Jahr, den Stock mit Silbergriff in der Linken, in der Rechten die sorgsam behütete Meerschaumpfeife - einen echten Schwanenhals! - in sein Café und in sein Stammgasthaus, immer noch auf seinem Grund ein angesehener Bürger, „der halt vom Seinigen lebt“ $[\ldots]$. (ÜB, 184)

Doch die Antiquiertheit dient wie das Fehlen wirtschaftlicher und sozialer Mobilität nur als ein Nebenmotiv, ${ }^{47}$ „Moraldarwinismus“ (Gauß) besiegelt den Untergang der Familie. Mayer bleibt „reichlich breit, aufgeblasen und wienerisch frohmütig “ 48 , auch wenn die Familie längst die Firmenleitung abgegeben, den Familiensitz in ein Zinshaus umgewandelt hat und beim Greißler aufschreiben lassen muss. Die Bezirksgrenzen wagt er, der „auf seinem Grund“ einem Monarchen gleicht, kaum zu überschreiten, selbst als er die Politik als Betätigungsfeld entdeckt. So wird rasch deutlich, dass er und sein Sohn Adam jun. - „Im günstigsten Fall ein schlimmerer Franz Mayer, oder noch was viel, viel Böseres" (ÜB, 241), urteilt die Mutter prophetisch - als unzeitgemäße, bildungsfeindliche wie unflätige Typen sich nicht mehr dazu eignen, der Familie zu altem Glanz zu verhelfen. Adam, vom Vater mit dem „bequeme[n] Fatalismus“ der Devise „[E]r geht net unter“ (ÜB, 230) erzogen und primär dem Alkohol und sexuellen Ausschweifungen zugewandt, ist schließlich für den Tod der mysteriösen, als dea ex machina der spätrealistischen Literatur verhafteten Ahnfrau Eva, ebenfalls im Haus wohnenden zweiten Ehefrau des Firmengründers („Eine Gestalt aus der Vergangenheit.“ - ÜB, 219), verantwortlich. Sie stirbt, von Adam bedrängt, vor Schreck. Mit gestohlenem Geld zieht Adam mit dem Hausmädchen Resi ein letztes Mal in ein verruchtes Tanzlokal am Stadtrand („Hier war er König. Hier galt er immer noch.“ - ÜB, 370), wo er im Streit von seinem einzigen Freund erstochen wird. „Dann erloschen die Lichter. Der Tanz

47. Daher erscheint der Vorschlag Wolfgang Beutins, den Roman als antikapitalistisch zu lesen, überzogen. Vgl. W. B., „Subversive Potentiale in den Dichtungen Jakob Julius Davids“, in Johann Dvořák (Hg.), Radikalismus, demokratische Strömungen und die Moderne in der österreichischen Literatur. Frankfurt am Main [u.a.] (= Bremer Beiträge zur Literatur- und Ideengeschichte, Bd. 43), P. Lang, 2003, S. 175-196, hier S. 180; Vgl. dazu auch Krappmann, Übergänge ..., a.a.O., S. 260-262.

48. Eugen Kalkschmidt, „Der Uebergang. Roman von J. J. David“, Kunstwart 17 (1904), H. 14 , S. 79. 
war zu Ende." (ÜB, 379). Am Leichnam des Sohnes mit den Vorwürfen seiner Frau konfrontiert („Was tust noch auf derer Welt? Zugrund zu richten hast nix mehr. Was willst noch, Franzl?" - ÜB, 391f), erhängt sich der Patriarch.

Zeitgemäße Mobilität zeigen hingegen die Frauenfiguren, die unterschiedliche weibliche Lebensentwürfe um 1900 ausfüllen und sich mit ihrer Entscheidungsautonomie auch dem Davids Texte kennzeichnenden Determinismus entziehen. Mutter Kathi sen., die, als Individuum aufgegeben, mit einem Wäschegeschäft den Hausstand finanziell erhält, blickt über die Ära ihres Gatten hinaus. „Es war ja gewiß: sie lebten im Übergang. Aber das mußte wo auf festem Boden sein Ende nehmen. Dem kommenden Geschlecht mochten bessere Zeiten beschieden sein." (ÜB, 240) Die erfolgreichen Ausbrüche der drei Töchter aus dem patriarchalischen System verlaufen höchst divergent: Die älteste Tochter Kathi, der als Eigenschaften bloß Schönheit und Geistesabwesenheit zugeschrieben werden (vgl. ÜB, 214f), brennt mit einem Adeligen durch, während Resi und Linnerl durch Fleiß ihr Glück erreichen. Resi, intellektuell benachteiligt und von der Arbeit für das mütterliche Geschäft gezeichnet, ergreift unterstützt von der Ahnfrau mit dem vom Vater polternd abgelehnten slawischen Tischlergesellen Xaver Navratil die Gelegenheit, über das Handwerk wieder zur angesehenen Bürgerin zu werden. ${ }^{49}$ Und Linnerl, der Jüngsten, wird vom Erzähler die Ausflucht in die Bildung (mit unklarem Ausgang) gestattet, ohne als Karikatur der bürgerlichen Frauenbewegung herhalten zu müssen. Sie entkommt nach der heimlichen Landpartie nach Heiligenkreuz auch dem „männlichen Domestikationswillen " ${ }^{50}$ des Hauslehrers Gröger, dem anpassungswilligen Studenten aus Reichenberg. „Ich möcht' lernen. Viel lernen. Was es für unsereins nur zum lernen gibt. [...] Vielleicht auf eine Lehrerin möcht' ich lernen. Und ich werd' dir's nie vergessen, daß du mir den Geschmack da darauf bracht hast.“ (ÜB, 395)

49. Dass eine Schwangerschaft als Druckmittel für die Zustimmung des Vaters dienen soll, bringt Resi zunächst in arge moralische Nöte. „[D]a ist das vierte Gebot. Und das steht freilich, und dawider ist nichts zu tun." (ÜB, 252) Die deutlichen Anklänge an Ludwig Anzengruber erinnern an die intensive Auseinander Davids mit dem Autor, dem er 1904 eine Monographie widmete. Später bezeichnete Erwin H. Rainalter David als „Anzengruber des Romans“ - Vgl. E. H. R., „Jakob Julius David. Zu seinem 20. Todestage", Neues Wiener Tagblatt, 20.11.1926, S. 6.

50. Krappmann, Übergänge ..., a.a.O., S. 259. 


\section{Unabgeschlossene Gegenentwürfe zum Wien der Jahrhundertwende}

Mit den beiden Romanen gelang es David, sich trotz des „Kesseltreiben[s] der Bahr-Clique ${ }^{\text {“51 }}$ als "Geschichtsschreiber des neuen Wien “ ${ }^{\text {52 }}$ im literarischen Feld zu positionieren. Wie er es Zola attestierte, reißt auch David unter der schillernden Oberfläche liegende Realitäten des urbanen Lebens „mit brutaler Faust in all ihrer armen Nacktheit in den grellen $\mathrm{Tag}^{\text {"53 }}$, läuft damit kulturhistorischen Imaginationen des von Carl E. Schorske geprägten Komplexes, Wiens um 1900' deutlich zuwider und verbreitert auch mittels naturalistischer Verfahrensweisen den literarischen Blick auf die Wiener Gesellschaft der Zeit. Schon nach Am Wege sterben schrieb Anton Lindner, Kritiker des Neuen Wiener Journals, in einem Brief:

Daß Sie durch Ihr Buch den modernen Wiener Problemroman geradezu inaugurieren [...] - das wird man, denk' ich, jetzt allgemein einräumen müssen. Dennoch wird man, weil man nicht zwischen den Zeilen zu lesen vermag, auch künftighin den stumpfsinnigen Ruf nach dem, Wiener Roman, der noch nicht geschrieben ist', nicht einstellen. Zwischen den Zeilen Ihres Buches aber steht, daß kein anderer als Sie berufen ist, diesen Wiener Roman zu bilden, denn ein Wiener wird ihn nie zustande bringen. Dazu gehören zwei kräftige Könnerfäuste, die aus dem bäuerlichen Nachbarboden in die Stadt hinübergreifen und sie zu ihren Zwecken formen. ${ }^{54}$

Davids niemals überwundene Distanz zur, Wiener Schule wird bei Lindner wie auch später bei Holzer ${ }^{55}$ ins Positive gewendet, sei gerade der ewige Fremde dazu berufen, die urbanen Realitäten zu erfassen, in jenem Sinne, in dem später Walter Benjamin das Wesen der Stadtbeschreibung umreißen sollte. ${ }^{56}$ Doch Davids Griff nach der Stadt

51. Jakob Julius David an Max Halbe, 22.4.1898, zitiert nach Groeneweg, Verhältnis, a.a.O., S. 194.

52. Richard Maria Werner, „J. J. David. Ein Erinnerungsblatt“, in Westermanns Monatshefte 55 (1910), H. 4, S. 343.

53. Jakob Julius David, „Emil Zola“, in Neues Wiener Journal, 30.9.1902, S. 1-2, hier S. 1.

54. Anton Lindner an Jakob Julius David, o. D., zitiert nach Peck, „Paralysis ...", S. 48.

55. Rudolf Holzer, „J. J. David“, Neues Wiener Journal, 17.11.1926, S. 3-4, hier S. 3.

56. Walter Benjamin, „Die Wiederkehr des Flaneurs“ [1929], in W. B., Gesammelte Schriften. Bd. III. Hg. von Hella Tiedermann-Bartels, Frankfurt/M., Suhrkamp, 1972, S. 194-199, hier S. 194. 
war nicht abgeschlossen, er gedachte eines Romanzyklus von „RougonMacquartscher Spannweite" ${ }^{\text {57 }}$. Mit Blick auf Goethes Wilhelm Meister forderte er, mit dem Roman „einen Abriß der Gesellschaft in ihrer Ganzheit und nach all ihren Verzweigungen [...] [zu] geben" ${ }^{\text {" } 58}$, in der Trilogie erkannte er „eine in der Natur selber gegründete Form“59. Ein geplanter dritter Wiener Roman mit dem verheißungsvollen Titel Die Sieger ließ sich jedoch nicht mehr realisieren.

57. Vgl. u.a. Eugen Schick, „Für J. J. David!“, Feuilleton-Beilage des Tagesboten aus Mähren und Schlesien 59 (1909), Nr. 14, S. 2, zitiert nach Krappmann, Übergänge ..., a.a.O., S. 247.

58. Jakob Julius David, „Goethe“, Neues Wiener Journal, 27.8.1899, S. 1-2, hier S. 2.

59. Jakob Julius David, „Vom Schaffen und seinen Bedingungen“, in J. J. D., Vom Schaffen. Essays, Jena, Diederichs, 1906, S. 106-166, hier S. 108. 